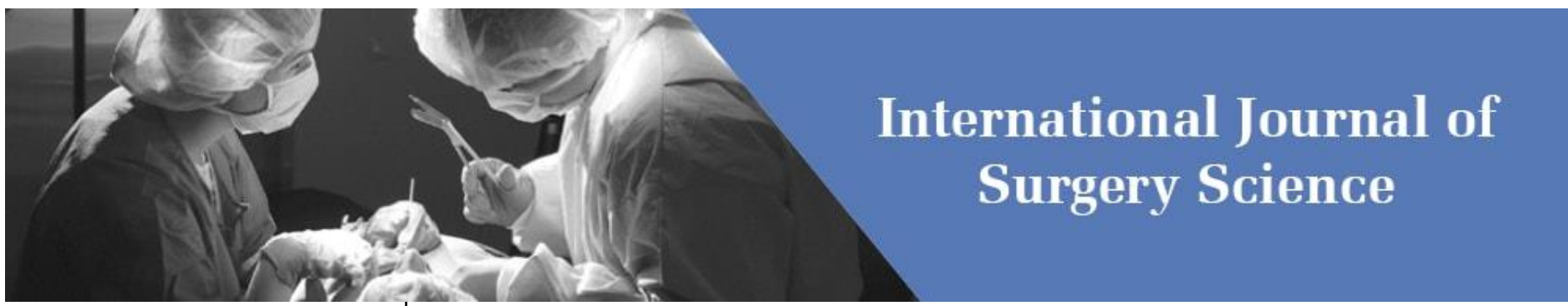

E-ISSN: 2616-3470

P-ISSN: 2616-3462

(C) Surgery Science www.surgeryscience.com

$2020 ; 4(4): 137-141$

Received: 07-08-2020

Accepted: 09-09-2020

Dr. Abbas Silman Altaei

Kerbala Health Directorate, Iraq

Dr. Abdulameer Raheem Hussein Kerbala Health Directorate, Iraq

Dr. Aamer Naji Shaalan

Kerbala Health Directorate, Iraq

\section{Screw fixation versus tension band wiring in Treatment of closed displaced horizontal oblique medial malleolar fracture}

\author{
Dr. Abbas Silman Altaei, Dr. Abdulameer Raheem Hussein and Dr. Aamer \\ Naji Shaalan
}

DOI: https://doi.org/10.33545/surgery.2020.v4.i4c.549

\section{Abstract}

Background: Fractures of the medial malleolus are part of the ankle injures which require an orthopedic care. Most of these injuries are low energy, rotational injuries. Many modalities of treatment are present, including non-operative or operative treatment to restore normal anatomical alignment and to resume normal fictional ability of the ankle.

Aim of study: To determine the outcome of surgical treatment of the medial malleolar fractures by tension band wiring versus screws fixation techniques.

Patients and Methods: Randomized controlled trial conducted to study two groups of patients at Department of Orthopedic surgery in Al- Hussain medical city teaching hospital from July 2017 to July 2020 , to evaluate the outcome of 36 patients with closed displaced horizontal oblique medial malleolar fractures. All the patients admitted to the emergency department of the hospital and operated within 24 hours. The study is based on 36 patients including males and females having closed displaced medial malleolar fractures of skeletally mature patients, divided in to two groups; group (A) managed by tension band wiring and group (B) with screws fixation, and followed for a maximum period of 20 weeks looking for union rate, infection, loss of reduction and the reoperation rate.

Results: Out of the (36) patients, follow up was missed in 2 patients, the other (34) patients were divided in to two groups (A\&B) and followed for a maximum period of 20 weeks. In groups A (16 parents with tension band wiring); The union rate was $(87.5 \%)$ within 12 weeks, delayed union (union achieved after 12 weeks and before 20 weeks) in 2 patients $(12.5 \%)$, no cases of non- union, infection reported in one patient (6.2\%), no case with reoperation had been reported. In groups B (18 patients with scores fixation), the union rate was $(77.8 \%)$ within 12 weeks, delayed union in 4 patients $(22.2 \%)$, no cases of nonunion, infection reported in one patient $(5.6 \%)$, and reoperation in two parents $(11.1 \%)$ had been reported.

Conclusion: From this study, we concluded that the results of both groups were good regarding the union rates, low risk of infection, and low reparation rate in screws fixation groups. The tension band wiring technique seems to be more rigid as a fixation technique with no reoperation rate.

Keywords: Medial malleolar fractures, tension band wiring, screw fixation

\section{Introduction}

Fractures of the ankle are among the most common injuries requiring an orthopedic care. Risk factors for sustaining an ankle fractures include age, increased body mass, and a history of ankle fractures. The highest incidence of ankle fractures occurs in elderly women. Isolated malleolar fractures account for two-thirds of ankle fractures ${ }^{[16]}$. Most ankle fractures are low-energy, rotational injuries, and these injuries cause destruction of not only the bony architecture, but also of tern the ligamentous and soft-tissue components ${ }^{[1,16]}$. Medial malleolar fractures may result from direct impaction of the talus or from tension as the talus rotates or moves laterally following the fibula ${ }^{[1]}$. Fractures with significant axial loading mechanism are more sever and often result in tibia plafond fractures ${ }^{[16]}$. Closed medial malleolar fractures can be either vertical fracture, horizontal- oblique fracture, comminuted fracture, or avulsion fracture of the tip of the medial malleolus ${ }^{[1]}$. Many modalities of treatment are present, including closed reduction and casting or open reduction and internal fixation by either cancellous screws, cannulated screws, fully threaded screws, tension band wiring or plates and screws ${ }^{[1]}$.
Corresponding Author: Dr. Abbas Silman Altaei Kerbala Health Directorate, Iraq 
Surgical anatomy of the medial malleolus ${ }^{[13,16]}$ : The medial malleolus is the medial projection of the distal end of the tibia, and has larger surface anteriorly than posteriorly. The posterior border of the medial malleolus includes the groove for the posterior tibia tendon ${ }^{[13]}$. The medial malleolus includes the anterior colliculus, which is larger and extends approximately $0.5 \mathrm{~cm}$ distal to the smaller posterior colliculus, The deltoid ligament provides medial ligamentous support of the ankle. The important deep component of the deltoid ligament arises from the inter- follicular groove and posterior colliculus. The deep layer of the deltoid ligament is ashsort, thick ligament inserting on the medial surface of the talus ${ }^{[13]}$. The superficial deltoid ligament arises from the anterior colliculus of the medial malleolus and consist of three parts; talo-tibial, naviculo-tibial, and calcaneo-tibial bands, the blood supply to the medial malleolus is from malleolar branches of the anterior and posterior tibial arteries. The ankle joint is very strong during dorsi-flexion because it is supported by powerful ligaments and is crossed by several tendons that are tightly bound down by thickenings of the deep fascial called retinacula. Its stability is also greatest in dorsi-flexion because in this position the trochlea of the talus fills the mortise formed by the malleoli. The malleoli grip the talus tightly as it rocks anteriorly and posteriorly during movements of the ankle joint. The grip of the malleoli on the trochlea of the talus is strongest during dorsi-flexion of the foot because this movement forces the anterior part of the trochlea posteriorly, spreading the tibia and fibula slightly apart. This spreading is limited by the strong interosseous ligament and by the anterior posterior tibio-fibular ligaments that unite the leg bones. The ankle joint is relatively unstable during planterflexion. During this moment the trochlea of the talus moves anteriorly in the mortise causing the malleoli to come together. However, the grip of the malleoli on the trochlea is not so strong as during dorsiflexion.

Classification ${ }^{[1,7]}$ Medial malleolar fractures classified generally into closed and opened fractures, comminuted and noncomminuted, displaced and displaced fractures, comminuted and non-comminuted, displaced and displaced fractures [1]. Herscovici classification ${ }^{[7]}$ of medial malleolar fractures depend on the level and direction of fracture line

A. Tip avulsions (anterior colliculus).

B. Intermediate.

C. Level of plafond (horizontal- oblique).

D. Above plafond (Vertically oriented fractures).

Description of the screws ${ }^{[1]}$ : Screws are complex tools with a four- part construction: head, shaft, thread and tip. The head serves as an attachment for the screw driver. The head also serves as the counterforce against which compression generated by the screw acts on the bone. The shaft or shank is the smooth portion of the screw between the head and the threaded portion. The thread is defined by its root (or core) diameter, its thread (or outside) dimeter, its pitch (or distance between adjacent threads), and its lead (or distance it advances into the bone with each complete turn).The cross sectional design usually is buttress (ASIF screws) or V thread (usually used in machine screws).The tip of the screw is either round (requires petnapping) or self-tapping (fluted or trocar). The screws that cross the fracture line are referred to as an inter fragmentary screws

\section{Types of screws ${ }^{[1]}$}

1. Cortical screws: these are traded their entire length and are available in the following diameters: $4.5 \mathrm{~mm}, 3.5 \mathrm{~mm} 2.7$ $\mathrm{mm}, 1.5 \mathrm{~mm}$. the cortical scores can function as other positional or lag screws for inter fragmentary compression if the hole in the near cortex is over drilled.

2. Cancellous: these screws have larger thread that provide more purchase in soft cancellous bone and are more frequently used in the metaphyseal areas. The solid cancellous screws are availed in $6.5 \mathrm{~mm}$ and $4 \mathrm{~mm}$ diameters and in two thread length- $16 \mathrm{~mm}$ and $32 \mathrm{~mm}$.

3. Self-tapping, self-drilling screws: these are availed in the same sizes as cortical screws these screws have a small bit at the end of the screw to remove bone debris self-tapping screws have less pulled out strength because of their construction these screws are best used in external fixation pins.

4. Locking screws: these are self-tapping screws with a locking screw at the head these screws require precise predrilling to allow tight fixation with a locking plate.

\section{Preoperative evaluation}

A- Clinical evaluation [2, 16]: This should include general examination, description of the mechanism of injury, examination for deformity of the foot relative to the leg and the direction of any displacement to the foot (if present) should be performed. The condition of the skin must be considered. Soft tissue swelling should be assessed because it will affect surgical timing. The complete circularity and neurological examination should be done. The clinical evaluation should also include and evaluation of medical comorbidities of the patient (if present) especially diabetes mellitus.

B- Radiographic evaluation ${ }^{[1,16]}$ : The standard radiographs of the ankle include mortise, AP, and lateral views. The Ottawa ankle rules assist physicians to decide when it is appropriate to obtain radiographs in adults with ankle injuries. These guidelines are sensitive for ankle fracture, and they reduce the number of radiographs taken, along with associated cost. According to Ottawa rules, ankle radiographs are needed only if there is pain near the malleoli and one or more of the following conditions is present:-

- Age 55 years or older.

- Inability to bear weight.

- Bone tenderness at the posterior edge or tip of either malleolus.

The mortise view is obtained by internal rotation of the patient's leg by approximately 15 degrees such that the $\mathrm{X}$-ray beam is perpendicular to the trans-malleolar axis, The AP radiograph is obtained with the x-ray beam in line with the second ray of the foot. The subchondral bone of the tibia and fibula should form a continuous line around the talus on all views. The talo-crural angel (the angle between alien drawn perpendicular to the distal articular surface of the tibia and alien connection the lateral and medial malleoli) should be $85 \pm 4$ degree or within 5 degree of the control-lateral ankle on the mortise view. The medial clear space (the distance between the medial articular surface of the medial malleolus and the talar dome) should be less than or equal to $4 \mathrm{~mm}$ and should be equal to the superior clear space between the talus and the distal tibia on the mortise view, The tibio- fibular clear space (the distance between the medial wall of the - fibula and the tibial incisura surface) should be less than or equal to $6 \mathrm{~mm}$ on the mortise view.

\section{Operative guide lines}

At the time of surgery, perioperative antibiotics are required, 
placing the patient supine on the operating table, positioning of the injured leg in slight external rotation to expose the medial malleolus well, and a pneumatic tourniquet to assist with visualization of the surgical field are helpful ${ }^{[16]}$. With any approach, we must handle the skin with car, reflection the flap intact with its underlying subcutaneous tissue. The blood supply to the skin of this area is poor, and careful handing is necessary to prevent skin sloughing. We must also protect the grater saphenous vein and its accompanying nerve ${ }^{[1]}$.

\section{Surgical approaches ${ }^{[1]}$}

There are three main surgical approaches to the medial side of the ankle,

\section{A: Anteromedial approach [Colonna and Ralston]}

- Begin the incision at appoint about $10 \mathrm{~cm}$ proximal and 2.5 $\mathrm{cm}$ posterior to the medial malleolus and curve it interiorly and inferiorly across the center of the medical malleolus and inferiorly and posteriorly $4 \mathrm{~cm}$ toward the heel.

- Divide the flexor retinaculum and retract the flexor halluces longus tendon and the neuro- vascular bundle posteriorly and laterally, retract the tibilalis posterior and flexor digit rum longus tendons medially and anteriorly.

\section{B: Posteromedial approach [Broom head]}

- The line of approach lies midway between the posterior border of the tibia and the medial border of the Achilles tendon, curves inferior to the medial malleolus to the medial border of the foot.

- This approach permits expose of medial and posterior malleolus.

\section{C: Transverse medial approach [Koenig and Schaefer]}

- Curve the incision just proximal to the medial malleolus, expose the fracture site, reduction and fixation.

- It is not a popular method because despite utmost care, it is possible to injure the tibia vessels and nerve.

\section{A. Transverse approach. \\ B. Poster- medial approach. \\ C. Antra- medial approach surgical techniques}

\section{A. Screw fixation technique ${ }^{[1]}$}

- With a bone holding clamp or towel clip, bring the detached malleolus into normal position, and while holding it there internally fix it with two $2 \mathrm{~mm}$ smooth krieschner wires drilled across the fracture site perpendicular to fracture line as temporary fixation devices, Check the fracture reduction with fluoroscopic imagine, if the reduction is satisfactory, remove one of the Krischner wires, and insert a $4 \mathrm{~mm}$ lag screw, then remove and replace the other Krishchner wire.

- Carefully inspect the interior of the joint particularly at the supper medial corner to ensure the screw has not crossed the articular surface.

- Make fluoroscopic image to verify the position of the screws and the fracture.

\section{B-Tension band wiring technique ${ }^{[3]}$}

- Bone fragment is held in reduced position with bone holding clamp or towel clip.

- Two 0.45 Krischner wires are driven through the deltoid ligament and tip of medial malleolus and across the fracture sit, but not into the proximal tibial cortex.

- Tension band figure of 8 wire (20 gauge) can be anchored proximal through and anterior to posterior drill hole in metaphysis.
- 20 gauge wire is then passed around the Krischner wires and tightened in a figure of 8 fashion, The Krischner wires are cut and turned medially and then tapped into the bone.

\section{Rehabilitation ${ }^{[16,1]}$}

- The ankle in is immobilized in a posterior plaster splint with the ankle in neutral position and elevated.

- If the bone quality is good, and fixation is secure, the splint can be removed in 2 to 4 days and replaced with a removable splint or fracture boot, range of motion exercises are begun,

- Weight bearing is restricted for 6 weeks, after which partial weight bearing can be started if the fracture is healing well. Full weigh bearing is allowed after 12 weeks.

- If skin conditions, bone quality or other factors have prevented secure fixation, the fracture must be protected longer, the patient is placed in either a short leg or a long leg ono-weight bearing cast.

- It can be converted to a short leg cast in 4 to 6 weeks the patient is not allowed to bear a weight on the ankle until fracture healing is progressing well (8 to 12 weeks) a short leg walking cast is worn, and weight beating is progressed the cast is removed when the fracture has united.

\section{Patients and methods}

This study had been done at the orthopedic unit in Al-Hussain medical city teaching hospital from July 2017- Juley 2020, it involves 36 patients, 22 males and 14 females with closed displaced horizontal - oblique medial malleolar fractures, were operated on within 24 hours, meanwhile were immobilized by back slab temporarily (till the time of operation).Two patients were missed during follow up, the remaining were 34 patients, there were 22 patients $(64.7 \%)$ males and 12 patients $(35.3 \%)$ females, the mean age was 34.2 years (ranging from 22- 54 years),

Preoperative preparation: General assessment of the patients includes taking history of the accident, past medical history especially for diabetes mellitus (no patient), general examination, local examination for soft tissue swelling (all the patents were with mild swelling), regional exemption for nervure vascular status. Investigations had been done for every patients including good quality radiographs. PCV, biochemical investigations, viral hepatitis screen, three of the patients were with anemia requiring crossed matched blood transfusion preoperatively. Back slab had been applied for every patient, the involved limb is elevated ant preoperative antibiotic (ceftriaxone injection vial $1 \mathrm{gm}$ ) given about 1 hour before operating.

\section{Surgical procedures}

Under general anesthesia and patient at supine position, tourniquet applied, and the involved limb directed in external rotation. All the patients (whether treated with tension band wiring or with cancellous lag screws) had been approached with the antero-medial curved approach: -

- The incision began at a point about $10 \mathrm{~cm}$ proximal and 2.5 $\mathrm{cm}$ posterior to the medial malleolus and curve it anteriorly and inferiorly across the center of the medial malleolus and inferiorly and posteriorly $4 \mathrm{~cm}$ toward the heel.

- Dividing the flexor retinaculum and retraction of the flew or halluces longus tendon and the neurovascular bundle posteriorly and laterally, retraction the tibialis posterior and flexor digitiorum longus tendons medially and anteriorly.

- Exposing the medial malleolus by reflecting the periosteum with preservation of the deltoid ligament. 


\section{A- Tension band wiring}

- After exposure of fracture site, reduction of fracture and holding the reduction with holding bone clamp or towel clip.

- Two Krishner wires were driven through the deltoid ligament and the tip of the medial malleolus and across the fracture site the orientation of the wires were nearly perpendicular to the fracture line.

- Anterior to posterior drill hole in metaphasis done and stainless steel wire anchored proximally through the hole and then passed around the Krischner wires and tightened in figure of 8 fashion.

- The Krishchner wires are cut, turned medially and then tapped into the bone.

- Average operative time was 70 minutes.

\section{B- Cancellous screw fixation}

- The fracture site exposed and reduced, then held temporarily with bone holding clamp or towel clip.

- Temporarily internal fixation done with two 2-mm smooth Krischner wires drilled across the fracture site and perpendicular to the fracture line.

- When the reduction is satisfactory, one of the Krischner wires removed and inspection of a $4 \mathrm{~mm}$ cancellous lag screw (measured so as not to cross the other tibial cortex). Then the other Krischner wire was removed and replaced by screw.

- Average operative time was 60 minutes.

\section{Post-operative follow up}

Short leg posteriors slab had been applied with the ankle in neutral position, elevation of the foot for 2-4 days till the pain and swelling subsided, then the slab was removed and replaced with a cast boot, removal of sutures after 2 weeks, the cast boot removed after 4 weeks, then range of motion exercises were begun. Weight bearing not allowed until the evidence of callus formation on radiographs (usually 4-6 weeks post operatively), then partial weight bearing started till the evidence of complete union on radiographs (usually after 12-14 weeks), then full weight bearing was adopted. All the patients were followed by a regular monthly visit with clinical and radiographic examinations for a maximum of 20 weeks follow up. There were 36 patients 2 of them were lost in the follow up, the remaining 34 patients (16 patients with tension band fixation and 18 patients with screws fixation) were followed for infection rate, time for radiological proved fracture unit ion, Hardwar failure, and loss of redaction.

\section{Results}

In this study open reduction and internal fixation were performed for 36 patients with clod displace horizontal - oblique medial malleolar fracture. Among them 34 parents $(94.4 \%)$ were followed to a maximum of 20 weeks and 2 patients $(5.6 \%)$ were lost during the follow up period.

\section{Criterial for estimating the results}

\section{Union rate}

- The fracture is regarded united if callus formation and union proven both clinically and radiologically.

- Delayed union id diagnosed when union is achieved after 12 weeks till 20 weeks.

- Nonunion: no clinical and radiological evidence of union till 20 weeks.

\section{Loss of reduction}

If there is articular step- off or gap more than 2-3 mm evident by radiological evaluation.

\section{Infection}

Classified as early (within 4 weeks), delayed (4-12 weeks) and late (after 12 weeks).

The result of group A (16 patients treated with tension band wiring) showed that bony union occurred in $14(87.5 \%)$ of the cases within 12 weeks, delayed union (more than 12 weeks) was seen in 2 patients $(12.5 \%)$ and nonunion cases not reported, Infection occurred in 1 patient $(6.2 \%)$ and was superficial early wound infection resolved well with oral antibiotics, no delayed or late wound infection was reported.

There was no case with loss of reduction or hardware failure. There was no need for reoperation, The results of groups B (18 patients treated with cancellous lag screws fixation) showed that bony union occurred in 14 patients $(77.8 \%)$ within 12 weeks, delayed union (more than 12 weeks) was seen in 4 patients $(22.2 \%)$, and nonunion cases not reported, Infection had been reported in 1 patient $(5.6 \%)$ and was superficial type of early wound infection resolved well with oral antibiotics, no delayed or late wound infection was present, There were 2 patients $(11.1 \%)$ presented after 6 weeks with hardware failure (screws pulled out) these 2 cases were treated with reoperation (removal of screws and adopting tension band wiring technique).

\section{Discussion}

This study was carried out on two methods (screw fixation and tension band wiring) of treatment of closed displaced medial malleolar fractures; the results were compared with the available data. In this study the union rate for tension band group was $(87.5 \%)$ less than reported in the study of Ostrom RF and Listky AS ${ }^{[17]}$ who reported $89 \%$. The delayed union rate $(12.5 \%)$ was more than $(11.6 \%)$ that reported in their study, the infection rate $(6.2 \%)$ was more than $(3.3 \%)$ which reported in their study. The union rate for screw fixation group was $(77.8 \%)$ which is less than reported in the study of Bostman and Pihljamake ${ }^{[1]}$ who reported $(92.7 \%)$. The infection rate $(5.6 \%)$ was more than $(4.3 \%)$ which reported in their study. Nonunion was reported in $(4.3 \%)$ in the study of Lauren Karger for fixation with partial herded lag screw, and there were $(23 \%)$ rate of lessening some of them require reparation for hardware removal ${ }^{[18]}$. In the study of Christy M. for screw fixation of medial malleolar fractures, there were $(91.3 \%)$ union rate and $(4.35 \%)$ nonunion rate also $(8.7 \%)$ rate for hardware loosening was reported ${ }^{[19]}$.

\section{Conclusions}

Rom this study we can conclude that these 2 techniques (tension band wiring and screw fixation) for the Tremont of closed displaced horizontal- oblique medial malleolar fracture have good results in union rates, lower infection rates, and low reoperation rate in screws fixation group.The tension band wiring technique seems to be more rigid as a fixation technique with no reparation rate.

\section{Recommendation}

1 hope that our respectful consultants or our colleagues will do a wide range of studies on this subject with larger number of patients to reach for the best methods for treatment of this type of medial malleolar fractures. 


\section{References}

1. Canale \& Beaty, Cambell's Operative Orthopedics, $11^{\text {th }}$ edition 2008;3087-3093:31-33.

2. Miller review of orthopedics $6^{\text {th }}$ edition 2012, 764-768.

3. Whee less' Textbook of orthopedics- AAOFAS, medial malleolar fractures 1996.

4. Robert RS. Surgical treatment of the displaced ankle fractures, Clin Orthop Relat Res 1983;172:164.

5. Physio Advisor physiotherapy for medial malleolar fractures 2008.

6. Park SS, Kubial EN, Egol KA et al. stress radiograph, after ankle fracture: the effect of ankle position and deltoid ligament status on medial clear space measurments, J orthop Trauma 2006;20:11.

7. Foot, Ankle Hyperbook, ankle fracture, medial malleolar fracture 2008.

8. East Lancashire Hospital NHS Trust 2010.

9. McDade WE: Diagnoses and treatment of ankle injuries, Instr Course Lect 1975;24:251.

10. Professional healt systems, copyright ankle - joint 2013.

11. Konrath G, Karges D, Watson JT et al. early versus delayed treatment of sever ankle fracture: a comparison of results, J Orthop Trauma systemic organization of the lower member anatomy Mod5, medial malleolus 1995;9:377.

12. Heide, David Nunn, copyright. The ankle, talus, and calcaneus, Normal radiographs of ankle 2009.

13. Clinically oriented anatomy, Keith L. Moore, second edition, chapter 4,541-543.

14. Medial malleolar fractures: A Biomechanical study of fixation techniques. T Ty Fowler, MD, Kevin J Pugh MD; Alan S Litsky MD.

15. Surgical exposures in orthopedics: The anatomic approach. Anterior and posterior approaches to the medial malleolus.

16. Comprehensive Orthopedic Review: fractures of the ankle, chapter 2009;60:659.

17. Ostrum RF, Listsky AS, Division of orthopedics, Ohio state university, Columbus 43210, Journal of orthopedic Trauma 1992;6(4):464-8.

18. Ricci WM, Tornetta P, Borrelli, Lag screw fixation of medial malleolar fractures, Journal of Orthopedic Trauma 2010;26(10):602-606.

19. A review of cases at Risk for Complicated Bone Healin 2012;51:39-44. 